\title{
Energy deposition studies for the high-luminosity Large Hadron Collider inner triplet magnets
}

\author{
N. V. Mokhov, ${ }^{*}$ I. L. Rakhno, and I. S. Tropin \\ Fermi National Accelerator Laboratory, Batavia, Illinois 60510, USA \\ F. Cerutti, L. S. Esposito, and A. Lechner \\ CERN, Geneva 23, CH-1211, Switzerland \\ (Received 6 March 2015; published 6 May 2015)
}

\begin{abstract}
A detailed model of the high-luminosity $\mathrm{LHC}$ inner triplet region with new large-aperture $\mathrm{Nb}_{3} \mathrm{Sn}$ magnets, field maps, corrector packages, and segmented tungsten inner absorbers was built and implemented into the FLUKA and MARS15 codes. Detailed simulations have been performed coherently with the codes on the impact of particle debris from the $14-\mathrm{TeV}$ center-of-mass pp-collisions on the shortand long-term stability of the inner triplet magnets. After optimizing the absorber configuration, the peak power density averaged over the magnet inner cable width is found to be safely below the quench limit at the luminosity of $5 \times 10^{34} \mathrm{~cm}^{-2} \mathrm{~s}^{-1}$. For the anticipated lifetime integrated luminosity of $3000 \mathrm{fb}^{-1}$, the peak dose calculated for the innermost magnet insulator ranges from 20 to $35 \mathrm{MGy}$, a figure close to the commonly accepted limit. Dynamic heat loads to the triplet magnet cold mass are calculated to evaluate the cryogenic capability. FLUKA and MARS results on energy deposition are in very good agreement.
\end{abstract}

DOI: 10.1103/PhysRevSTAB.18.051001

PACS numbers: 29.20.db, 84.71.Ba

\section{INTRODUCTION}

The Large Hadron Collider (LHC) was operated in 2012 at $4 \mathrm{TeV}$ per beam and $70 \%$ of the design luminosity of $10^{34} \mathrm{~cm}^{-2} \mathrm{~s}^{-1}$. After the 2013-2014 shutdown for accelerator improvements and maintenance, it will provide $300 \mathrm{fb}^{-1}$ of integrated luminosity at a center-of-mass (c.m.) energy of $13-14 \mathrm{TeV}$ by 2021. Subsequently, CERN is planning to make a high-luminosity upgrade (HL-LHC) to integrate at least $3000 \mathrm{fb}^{-1}$ of luminosity in ten years [1], with $4000 \mathrm{fb}^{-1}$ as the ultimate goal.

One essential objective of the HL-LHC upgrade is to reduce $\beta *$ down to $10-15 \mathrm{~cm}$ by means of stronger and larger aperture low-beta triplet quadrupoles in the highluminosity insertion regions (IRs). The envisaged solution [2] relies on the new $\mathrm{Nb}_{3} \mathrm{Sn}$ technology, which allows a more compact layout and $\sim 30 \%$ higher performance with respect to NbTi coils, and on a $150 \mathrm{~mm}$ aperture, doubling the present one of $70 \mathrm{~mm}$. In addition, a superconducting D1 separation dipole will replace the normal-conducting version. As well, new quadrupoles in the matching section are foreseen, still based on NbTi technology, but with a larger aperture.

From the quench stability and radiation damage points of view, these magnets should cope with an exceptionally high

\footnotetext{
*mokhov@fnal.gov

Published by the American Physical Society under the terms of the Creative Commons Attribution 3.0 License. Further distribution of this work must maintain attribution to the author $(s)$ and the published article's title, journal citation, and DOI.
}

luminosity. They need to be designed to operate at $\mathrm{L}=$ $5 \times 10^{34} \mathrm{~cm}^{-2} \mathrm{~s}^{-1}$ (corresponding to 5 times nominal LHC peak luminosity) or at an ultimate $\mathrm{L}=7.5 \times 10^{34} \mathrm{~cm}^{-2} \mathrm{~s}^{-1}$, with appropriate safety margins. Assumed design limits [3-5] are 13 and $4 \mathrm{~mW} / \mathrm{cm}^{3}$ for $\mathrm{Nb}_{3} \mathrm{Sn}$ and $\mathrm{NbTi}$ coils, respectively, including a safety factor of 3 on expected quench limits. For long term radiation damage, a tentative dose limit is set to a few tens of MGy, mainly because of the degradation of the epoxy resin used to impregnate $\mathrm{Nb}_{3} \mathrm{Sn}$ coils [6]. As the first studies of radiation loads in the LHC upgrades have shown [3,7], one could provide the operational stability and adequate lifetime of the inner triplet (IT) superconducting magnets by using tungsten-based inner absorbers in the magnets. Another constraint is given by the total heat load to be removed from the ensemble of the IT, corrector package $(\mathrm{CP})$ and $\mathrm{D} 1$ magnets by the cryogenic equipment.

This paper is divided into five parts. Section II is devoted to characterization of the pp-collisions at the LHC interaction points as a source of irradiation of the IR magnets and to the approach used to design the inner protection absorbers in the IT. Section III gives the details of the FLUKA and MARS models built to study the problem. In Sec. IV, the Monte Carlo calculations of 3D distributions of power density in the coils of the IT magnets and the dynamic heat loads are presented. These FLUKA and MARS predictions are in excellent agreement and are safely below the quench limits. The accumulated absorbed dose, neutron fluence and displacement per atom (DPA) values at the hottest spots of the IT magnets defining the radiation damage and lifetime of the IT components-considered 
as one of the critical elements in the design of the HL-LHC IT magnets-are described in detail in Sec. V. Section VI is devoted to engineering constraints worsening the ideal protection effectiveness and possible design changes that would reduce the radiation dose and improve component lifetimes.

\section{COLLISION DEBRIS AND TRIPLET MAGNETS}

\section{A. Characterization of the radiation source}

Proton-proton inelastic collisions taking place in the LHC inside its four detectors generate on average about 100 (120) secondary particles per a single proton-proton interaction of 3.5 (7) TeV beams, with substantial event-to-event fluctuations, as calculated with DPMJET-III [8]. The latter is used in this study as a primary pp-event generator. It reproduces within $10 \%$ the charged particle energy flow in the forward rapidity range measured by the $\mathrm{LHCb}$ experiment at $\mathrm{LHC}$ in $7 \mathrm{TeV}$ c.m. minimum bias collisions $[9,10]$. We expect the same level of the uncertainty in using DPMJET-III at 14-TeV c.m. energy that is quite adequate for the purpose of this study. Note that the most recent DPMJET version [10] agrees with the $7-\mathrm{TeV}$ c.m. CMS data within a percent accuracy; we are going to switch to that version in future studies. Moving away from the interaction point (IP), this multiform population evolves, even before touching the surrounding material, because of the decay of unstable particles (in particular neutral pions decaying into photon pairs). Figure 1 illustrates the composition of the debris at $5 \mathrm{~mm}$ from the point of a $14-\mathrm{TeV}$ c.m. collision, featuring a $\sim 30 \%$ increase in number of particles, due to aforementioned decays, and a clear prevalence of photons (almost one half) and charged pions ( 35\%).

Most of these particles are intercepted by the detector and its forward region shielding releasing their energy

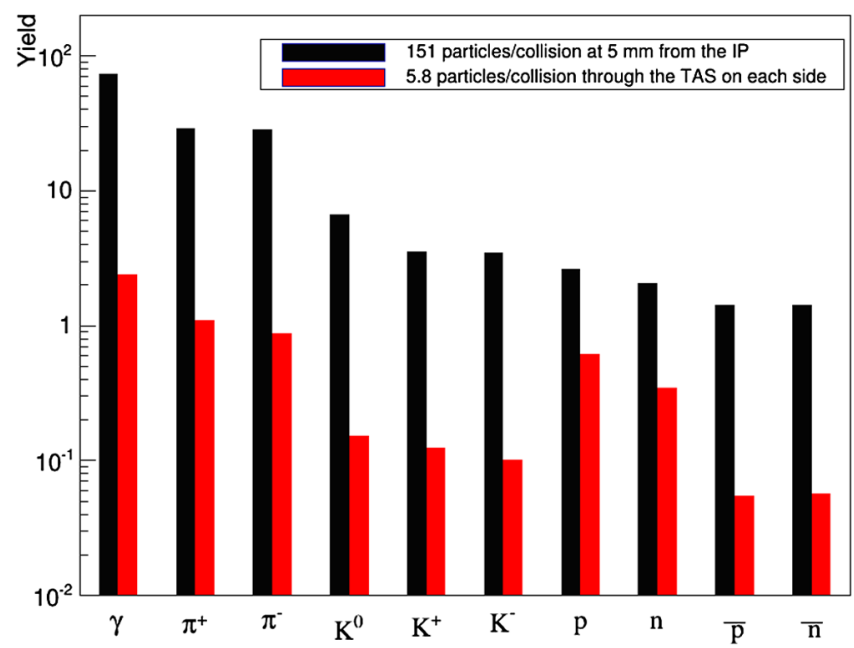

FIG. 1. Number of debris particles per single proton-proton inelastic interaction at $5 \mathrm{~mm}$ from the interaction point (black histogram) and at the exit of each 60-mm TAS aperture (red). within the experimental cavern. However, the most energetic ones, emitted at small angles with respect to the beam direction, travel farther in the vacuum and reach the accelerator elements, causing a significant impact on the magnets along the insertion regions, in particular the final focus quadrupoles and the separation dipole. Figure 1 shows also the breakdown of the debris components going through the $60-\mathrm{mm}$ aperture of the target absorber secondaries (TAS) absorber, a protection element consisting of a copper core $1.8-\mathrm{m}$ long located at $20 \mathrm{~m}$ from the IP and representing the interface between the detector and the accelerator. The TAS absorbers are installed at each side of the high-luminosity detectors, ATLAS in P1 and CMS in P5. Their protection role, in fact limited to the first quadrupole [11], is not needed for luminosities up to $0.2 \times 10^{34} \mathrm{~cm}^{-2} \mathrm{~s}^{-1}$ [12], which is the upgrade target of $\mathrm{LHCb}$.

Despite the fact that the number of particles per collision leaving the TAS aperture is more than 1 order of magnitude lower than the total number of debris particles, they carry about $80 \%$ of the total energy, implying that $40 \%$ of the released energy at the IP exits on each side of the experiments. At the nominal HL-LHC luminosity $\left(5 \times 10^{34} \mathrm{~cm}^{-2} \mathrm{~s}^{-1}\right)$, this represents about $3800 \mathrm{~W}$ per side that is inevitably impacting the LHC elements and consequently dissipated in the machine and in the nearby equipment (e.g., electronics, racks, ...) and in the tunnel walls.

It is fundamental to study how these particles are lost in order to implement the necessary protections for shielding sensitive parts of the LHC magnets and the machine. For these purposes, Monte Carlo simulations of the particle interaction with matter play an essential role, relying on a detailed implementation of physics models and an accurate $3 \mathrm{D}$ description of the region of interest.

\section{B. Large aperture $\mathrm{Nb}_{3} \mathrm{Sn}$ magnets and inner shielding}

The LHC upgrade includes replacement of the IP1/IP5 inner triplet $70-\mathrm{mm} \mathrm{NbTi}$ quadrupoles with the $150-\mathrm{mm}$ coil aperture $\mathrm{Nb}_{3} \mathrm{Sn}$ quadrupoles along with the new $150-\mathrm{mm}$ coil aperture NbTi dipole magnet and orbit correctors (MCBX1, MCBX2 and MCBX3). Moreover, a $\mathrm{CP}$ that includes a skew quadrupole and eight high-order magnets (from sextupole to dodecapole, normal and skew, based on the NbTi technology) will be located between the triplet and the D1.

An octagonal stainless steel beam screen, equipped with 6-mm tungsten absorbers on the midplanes, is placed inside the cold bore all along the triplet, the orbit correctors, the $\mathrm{CP}$ and the D1, except in Q1 (up to $\sim 32.5 \mathrm{~m}$ from IP) where the tungsten thickness is increased to $16 \mathrm{~mm}$, compatible with the relaxed aperture requirements. The absorbers are in between the beam screen and the 1.9-K beam pipe: they are supported by the beam screen, and thermally connected to it, whereas they have negligible contact with the cold mass. Therefore, from the point of 
view of energy deposition, the beam screen function is twofold: (i) it shields the coils from the debris by reducing the energy deposited in there; (ii) it removes a sizable part of the heat load from the $1.9 \mathrm{~K}$ cooling system, collecting it at higher temperature.

The present HL-LHC layout foresees six cryostats on each side of the IP: two for the first and last quadrupoles (with each quadrupole split in pairs: Q1 A-Q1B and Q3 A-Q3B), one for each half of the second quadrupole and its respective orbit corrector (MCBX1-Q2 A and Q2B-MCBX2), one for the MCBX3 and CP, and the last for the D1 dipole. The distance between the magnets in the interconnect regions is $1.5-1.7 \mathrm{~m}$ (as preliminarily assumed in this study) and an interruption of the beam screen is necessary therein. As a reasonable baseline, we adopt here a 500-mm interruption of the tungsten absorbers in the middle of the interconnect regions.

\section{MONTE CARLO MODELING WITH FLUKA AND MARS CODES}

To design such a system in a consistent and confident way, coherent investigations have been undertaken with two independent Monte Carlo codes benchmarked up to the $\mathrm{TeV}$ energy region and regularly used in such applications: FLUKA at CERN $[13,14]$ and MARS15 (2014) at Fermilab [15-17]. The studies were done for $7+7 \mathrm{TeV}$ pp-collisions at the luminosity of $5 \times 10^{34} \mathrm{~cm}^{-2} \mathrm{~s}^{-1}$ with a $0.295 \mathrm{mrad}$ half-angle vertical crossing in IP1 (which was found earlier $[11,18]$ to be the worst case) using DPMJET-III as the event generator.

An identical, very detailed geometry model was created and used in both codes with same materials and magnetic field distributions in each of the components of the $80-\mathrm{m}$ region from IP through the D1 dipole. Figures 2 thru 6

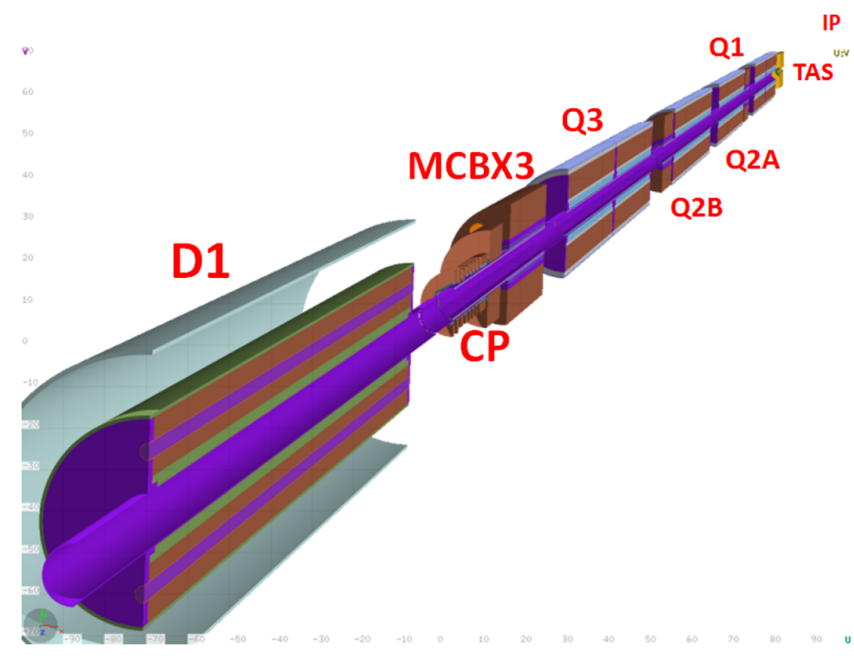

FIG. 2. Computer model of HiLumi LHC inner triplet with correctors $\mathrm{MCBX} / \mathrm{CP}$ and $\mathrm{D} 1$ dipole. show a 3D view of the model and details of the inner parts of the quadrupoles, orbit correctors and dipole D1.

In this study, $10^{5}$ to $2 \times 10^{5}$ nonelastic pp interactions at $14-\mathrm{TeV}$ c.m. energy generated with DPMJET-III are used in the FLUKA and MARS runs to typically provide a few percent statistical rms error in the finemesh gridded coil and other near-beam components of the IT magnets. The power density and dynamic heat load for each IT component are normalized to a luminosity of $5 \times 10^{34} \mathrm{~cm}^{-2} \mathrm{~s}^{-1}$, while the absorbed dose, neutron fluence and DPA are normalized to an integrated luminosity of $3000 \mathrm{fb}^{-1}$. Longitudinal scoring bins are $10 \mathrm{~cm}$, and azimuthal ones are $2^{\circ}$. Radially, power density is scored in the superconducting cable width, while dose, fluence and DPA are scored at the azimuthal maxima within the innermost layer equal to $3-\mathrm{mm}$ or its thickness, whatever is thinner.

\section{OPERATIONAL RADIATION LOADS}

Power density isocontours at the IP end of the cold mass of the Q2B quadrupole are shown in Fig. 7. The longitudinal peak power density profile on the inner coils of the IT magnets at the azimuthal maxima is presented in Fig. 8. Analyzing these two figures remember that the power density values are averaged radially over the first coil width (see Sec. III) to get the peak power density quantity for comparison with the quench limit. Results from FLUKA and MARS are in excellent agreement. The peak value in the quadrupoles, $2 \mathrm{~mW} / \mathrm{cm}^{3}$, is 20 times less than the quench limit of $40 \mathrm{~mW} / \mathrm{cm}^{3}$ in the $\mathrm{Nb}_{3} \mathrm{Sn}$ quadrupoles at $I_{\mathrm{op}} / I_{\mathrm{c}}=0.8[19,20]$. Here $I_{\mathrm{op}}$ is the magnet operational current, and $I_{\mathrm{c}}$ is the magnet critical current. The peak value of $\sim 1.5 \mathrm{~mW} / \mathrm{cm}^{3}$ in the $\mathrm{NbTi}$ based coils of the correctors and D1 dipole is almost 10 times less than the quench limit of $13 \mathrm{~mW} / \mathrm{cm}^{3}$ in such coils, again at $I_{\mathrm{op}} / I_{\mathrm{c}}=0.8$. Note that results of Refs. $[19,20]$ allow rescaling the quench limits to other values of $I_{\mathrm{op}} / I_{\mathrm{c}}$.

The total power dissipation in the IT region from the IP1 collision debris splits approximately 50-50 between the cold mass and beam screen with the tungsten absorber: 630 and $615 \mathrm{~W}$, respectively, from the FLUKA calculations. Total heat load to various components of the inner triplet, including comparison between FLUKA and MARS data, is presented in Table I. One can see that, as far as the total heat load is concerned, the two codes agree within about $2 \%$. For the 45-m effective length of the cold mass, the average dynamic heat load on it is $\sim 14 \mathrm{~W} / \mathrm{m}$. This is within a design range of $10-15 \mathrm{~W} / \mathrm{m}$ used for the LHC and assumed for the HL-LHC.

One can note that, if the assumed interruption of the shielding material along the interconnects (ICs) is reduced, resulting in a more extended absorber mass, the latter naturally collects more power while reducing the total in the cold masses. 
TABLE I. Integral power dissipation (W) in components of inner triplet calculated with FLUKA and MARS codes. FLUKA results are given for both 10 and $50 \mathrm{~cm}$ shielding gaps in the fixed-length ICs.

\begin{tabular}{|c|c|c|c|c|c|c|}
\hline \multirow[b]{3}{*}{ Component } & \multicolumn{4}{|c|}{ FLUKA } & \multirow{2}{*}{\multicolumn{2}{|c|}{$\frac{\text { MARS }}{50 \mathrm{~cm} \text { gap in ICs }}$}} \\
\hline & \multicolumn{2}{|c|}{$10 \mathrm{~cm}$ gap in ICs } & \multicolumn{2}{|c|}{$50 \mathrm{~cm}$ gap in ICs } & & \\
\hline & $\begin{array}{l}\text { Magnet cold } \\
\text { mass }\end{array}$ & $\begin{array}{l}\text { Beam } \\
\text { screen }\end{array}$ & $\begin{array}{l}\text { Magnet } \\
\text { cold mass }\end{array}$ & $\begin{array}{l}\text { Beam } \\
\text { screen }\end{array}$ & $\begin{array}{l}\text { Magnet } \\
\text { cold mass }\end{array}$ & $\begin{array}{l}\text { Beam } \\
\text { screen }\end{array}$ \\
\hline $\mathrm{Q} 1 \mathrm{~A}+\mathrm{Q} 1 \mathrm{~B}$ & 100 & 170 & 100 & 170 & 95 & 170 \\
\hline $\mathrm{Q} 2 \mathrm{~A}+$ orbit corrector & 95 & 60 & 100 & 65 & 100 & 65 \\
\hline $\mathrm{Q} 2 \mathrm{~B}+$ orbit corrector & 115 & 80 & 120 & 80 & 115 & 80 \\
\hline $\mathrm{Q} 3 \mathrm{~A}+\mathrm{Q} 3 \mathrm{~B}$ & 140 & 80 & 140 & 80 & 135 & 75 \\
\hline Corrector package & 55 & 55 & 60 & 55 & 60 & 65 \\
\hline D1 & 90 & 60 & 90 & 60 & 90 & 55 \\
\hline Interconnects & 20 & 140 & 20 & 105 & 15 & 85 \\
\hline Total & 615 & 645 & 630 & 615 & 615 & 600 \\
\hline
\end{tabular}

\section{LIFETIME RADIATION LOADS}

The peak dose and DPA - the quantities that define radiation damage and lifetime of insulators and nonorganic materials of the IT magnets, respectively - are calculated at the azimuthal maxima in the innermost tiny layers of each the IT component shown in Figs. 3-6.

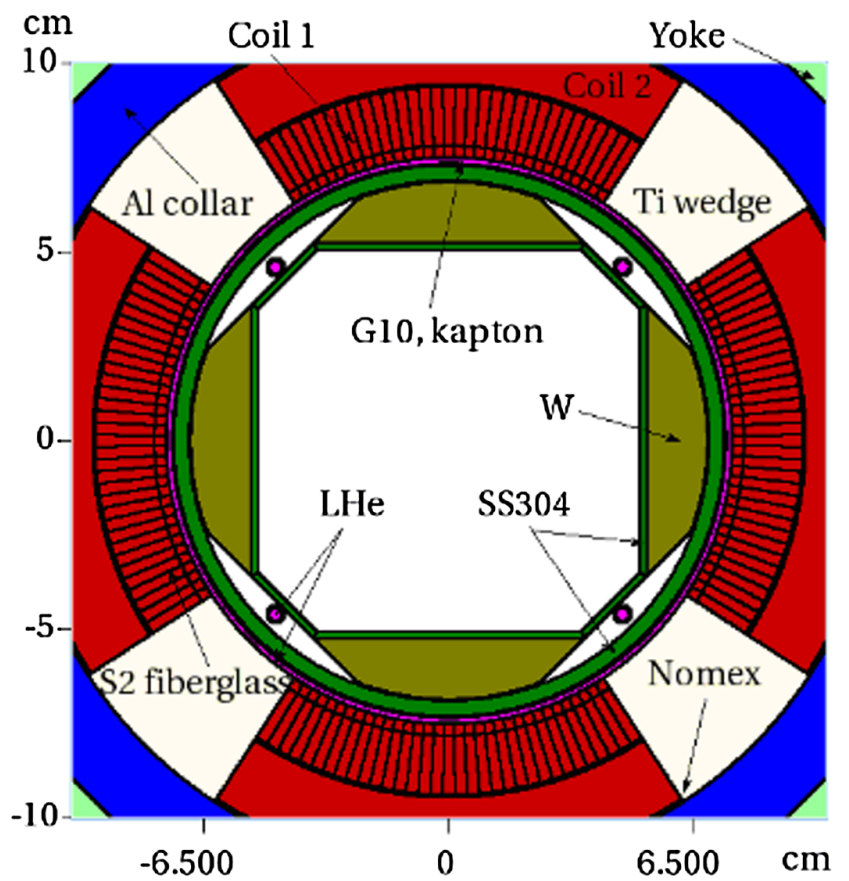

FIG. 3. Details of the FLUKA-MARS model in the innermost region of the Q1 quadrupole. The major difference between Q2-Q3 and Q1 is that the tungsten liner in the former is significantly thinner than that in the latter. The $\mathrm{Nb}_{3} \mathrm{Sn}$ coils are homogenized; inner coils are subdivided azimuthally and radially for scoring. Nomex and S2 fiberglass insulating inserts are not included in the model; corresponding labels indicate their locations as discussed in the text for Table II below.
The longitudinal peak dose profiles on the inner coils and insulating materials are presented in Fig. 9. The values in the MCBX orbit correctors in the Q1-Q2 A, Q2B-Q3 and Q3-D1 regions are given for the epoxy layer (FLUKA) and kapton layer (MARS); see Figs. 4-6 for details. Results from FLUKA and MARS are again in good agreement. The larger aperture IT magnets and the tungsten absorbers implemented perform very well, reducing the peak values of absorbed dose in the HL-LHC IT after $3000 \mathrm{fb}^{-1}$ to the level which is expected to be reached in the present LHC after $300 \mathrm{fb}^{-1}$ only. The ultimate goal of $4000 \mathrm{fb}^{-1}$ requires further work on the inner absorbers as well as on more radiation-resistant insulation to be used in the IT magnets.

The maximum peak dose in the coils is about 25 MGy for quadrupoles and $\sim 15 \mathrm{MGy}$ for the D1 dipole.

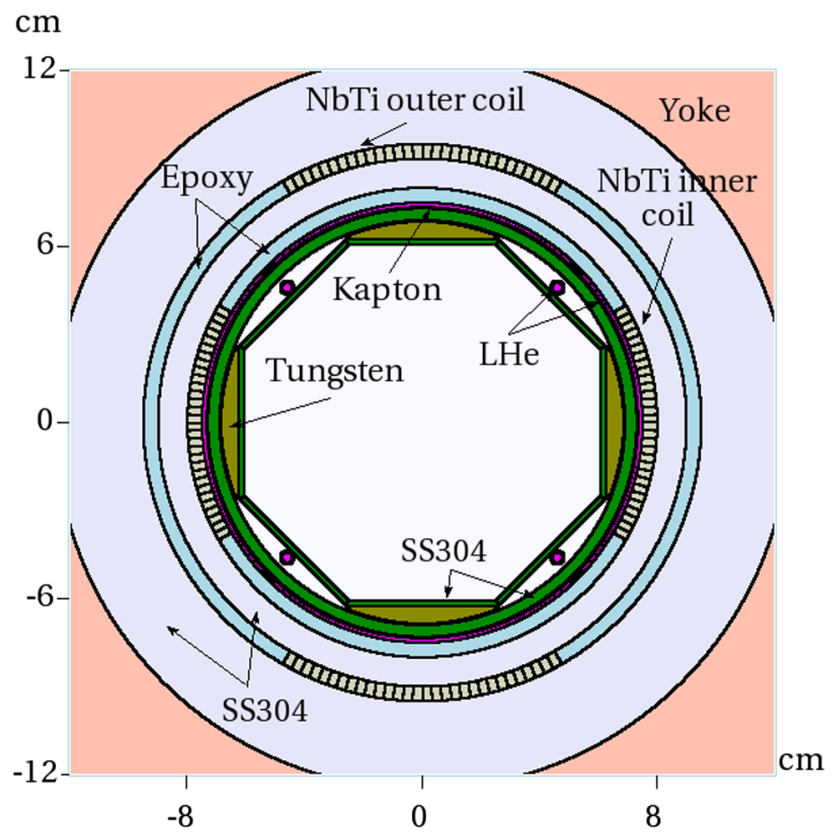

FIG. 4. Cross-sectional view of the FLUKA-MARS model in the central part of the MCBX orbit correctors. 


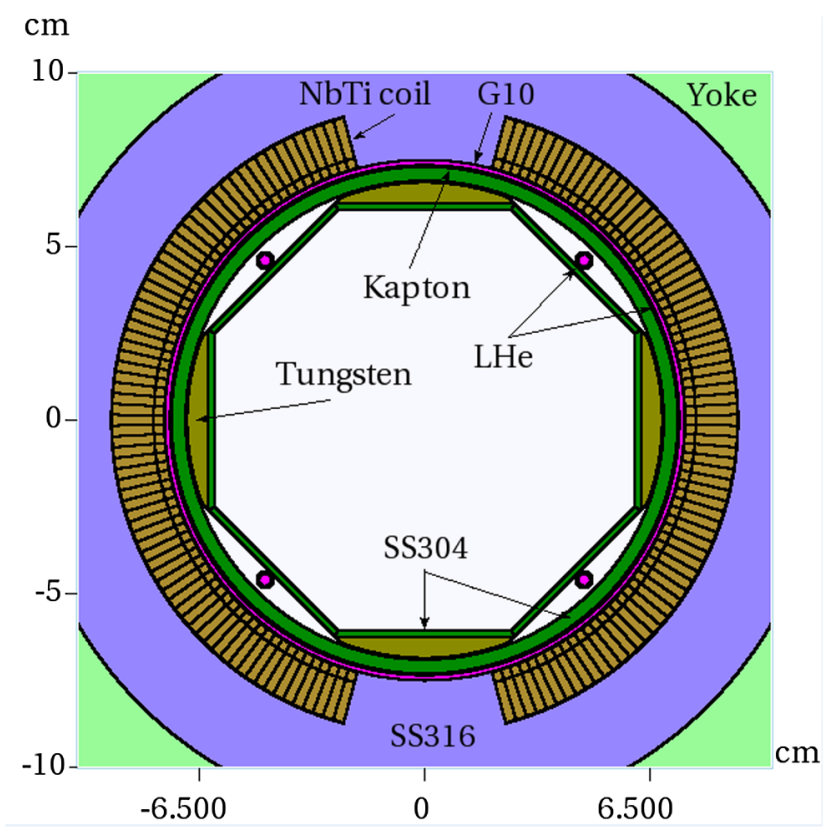

FIG. 5. Cross-sectional view of the FLUKA-MARS model in the central part of the D1 dipole. The coil is homogenized and subdivided azimuthally and radially for scoring.

The peak dose in the IT magnet insulation reaches 30-36 MGy in the MCBX3 corrector, 28-30 MGy in the quadrupoles and $22 \mathrm{MGy}$ in the D1 dipole (see Table II below). This is at the common limits [21] for kapton (25-35 MGy) and CTD-101 K epoxy (25 MGy) or slightly above them.

Another important issue is related to absorbed dose at the level of the beam screen. In order to mitigate electron

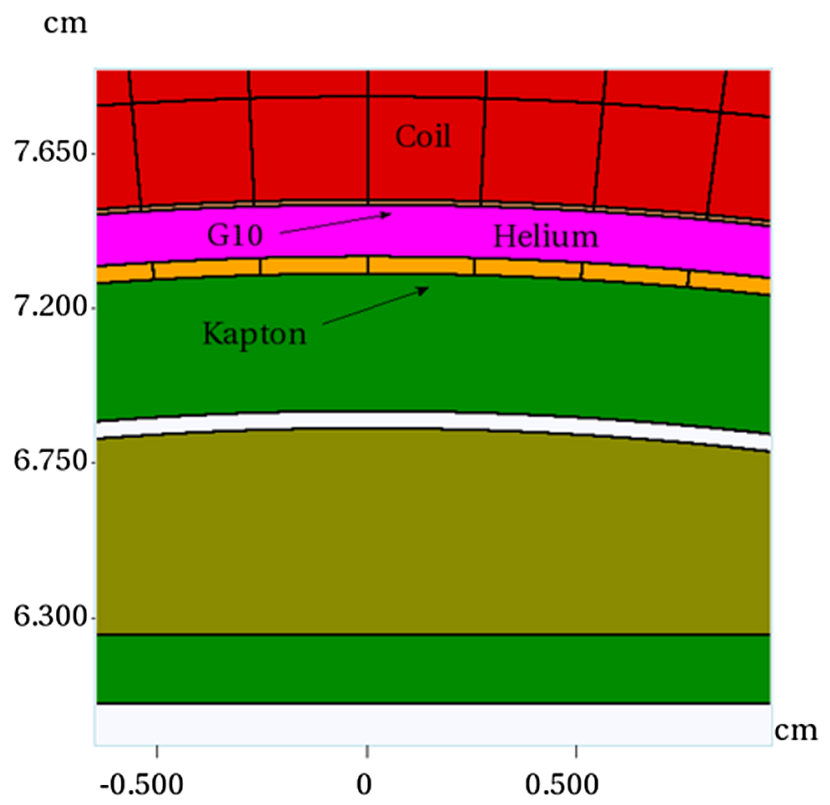

cloud heating [22], a thin-film carbon coating is deposited on the inner surface. Mechanical stability of the coating depends on various factors including irradiation. The calculated peak absorbed dose in the stainless steel beam screen is shown in Fig. 10.

Table II summarizes the peak predicted absorbed dose in the hottest components of the inner triplet. One can see that in the hottest spots of the triplet the calculated absorbed dose for the target integrated luminosity is near or slightly above the lifetime limit which means that degradation of material properties becomes relevant. One has to point out that the real magnets are more complicated than the simulation models built for this study. For example, some insulating inserts made of S2 fiberglass and Nomex as indicated, e.g., in Fig. 3, were not included in the model. Nevertheless, to estimate the radiation loads to these components, Table II includes the extrapolated peak dose values in such inserts using the calculated spatial dose gradients. That is why some peak values in Table II do not correspond to the data shown in Fig. 9.

Degradation of the critical properties of inorganic materials of the IT magnets $-\mathrm{Nb}_{3} \mathrm{Sn}$ and NbTi superconductors, copper stabilizer and mechanical structuresis usually characterized not by absorbed dose but by integrated neutron fluence and by DPA accumulated in the hottest spots over the expected magnet lifetime. DPA is the most universal way to characterize the impact of irradiation on inorganic materials. In both FLUKA and MARS, all products of elastic and inelastic nuclear interactions as well as Coulomb elastic scattering (NIEL) of transported charged particles (hadrons, electrons, muons and heavy ions) from $\sim 1 \mathrm{keV}$ to $\mathrm{TeV}$ energies contribute to

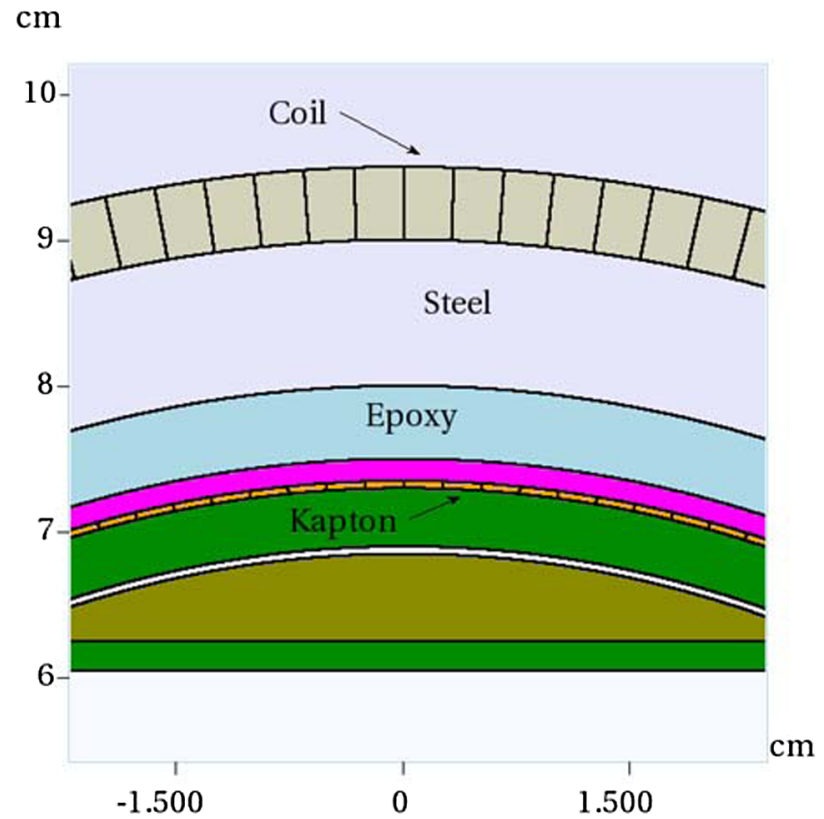

FIG. 6. Cross-sectional view of the FLUKA-MARS model fragments with kapton cells in Q1-Q3 quadrupoles (left) and MCBX correctors (right). 


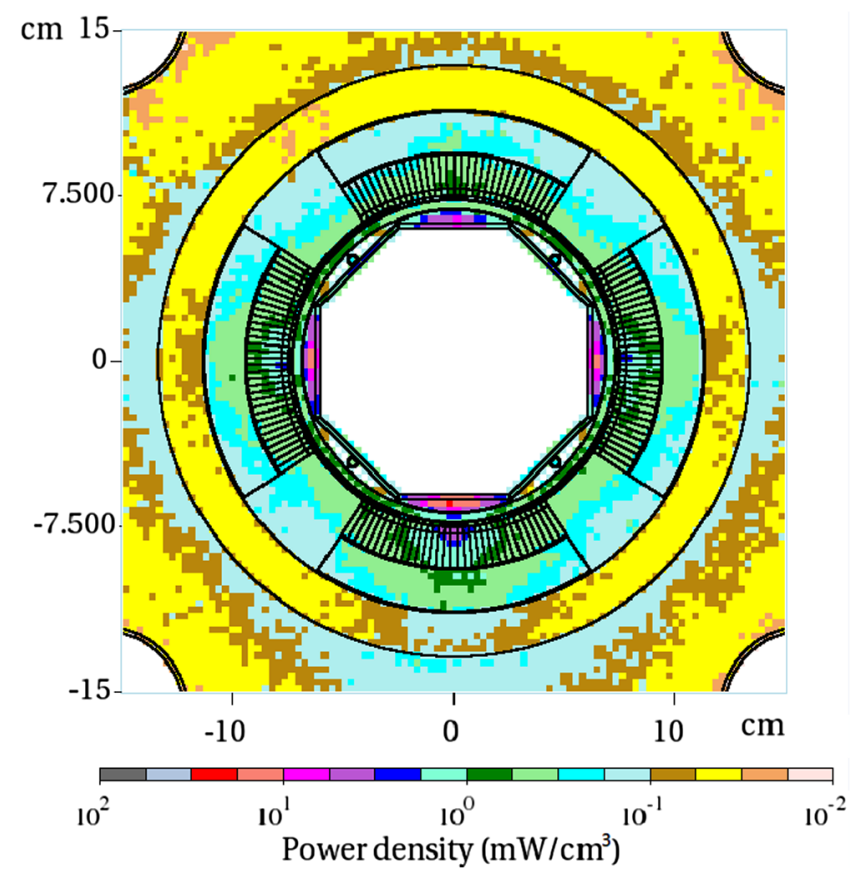

FIG. 7. Power density isocontours at the IP end of the Q2B quadrupole.

DPA using energy-dependent displacement efficiency. For neutrons with energies below $20 \mathrm{MeV}$ (FLUKA) and below $150 \mathrm{MeV}$ (MARS), the ENDF/B-VII database with NJOY99 [23] processing is used in both the codes.

The longitudinal peak neutron fluence and peak DPA profiles on the IT magnet coils are presented in Fig. 11. The peaks are generally observed at the inner coils; therefore, the data is given there. With the vertical crossing in IP1, the MCBX3 orbit corrector is the exception with the peak in the outer coil in the vertical plane (see Fig. 4). To see this effect, the MARS data in Fig. 11 for MCBX3 is given for the outer coil, while FLUKA shows results for the inner coil as in all other magnets.

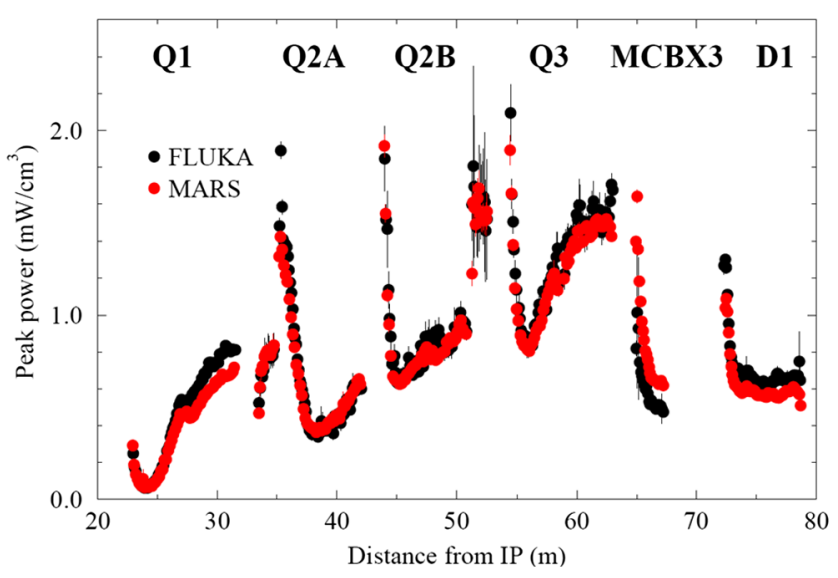

FIG. 8. Longitudinal peak power density profile on the inner coils of the IT magnets.

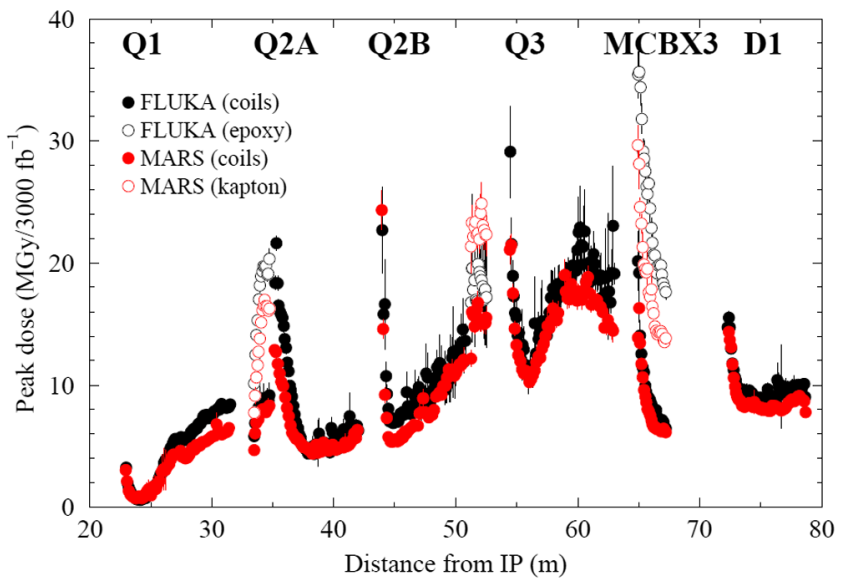

FIG. 9. Longitudinal peak dose profile on inner coils and nearby insulators.

Contrary to the power density and dose distributions driven by electromagnetic showers initiated by photons from neutral pion decay, DPA peaks at the non-IP end of the Q1B quadrupole. At that location, about $70 \%$ of DPA is from neutrons with kinetic energy below $20 \mathrm{MeV}, \sim 25 \%$ from transported nuclear recoils with the energy above $0.25 \mathrm{keV}$ per nucleon, and the rest is due to other transported particles and nontransported recoils. One can also
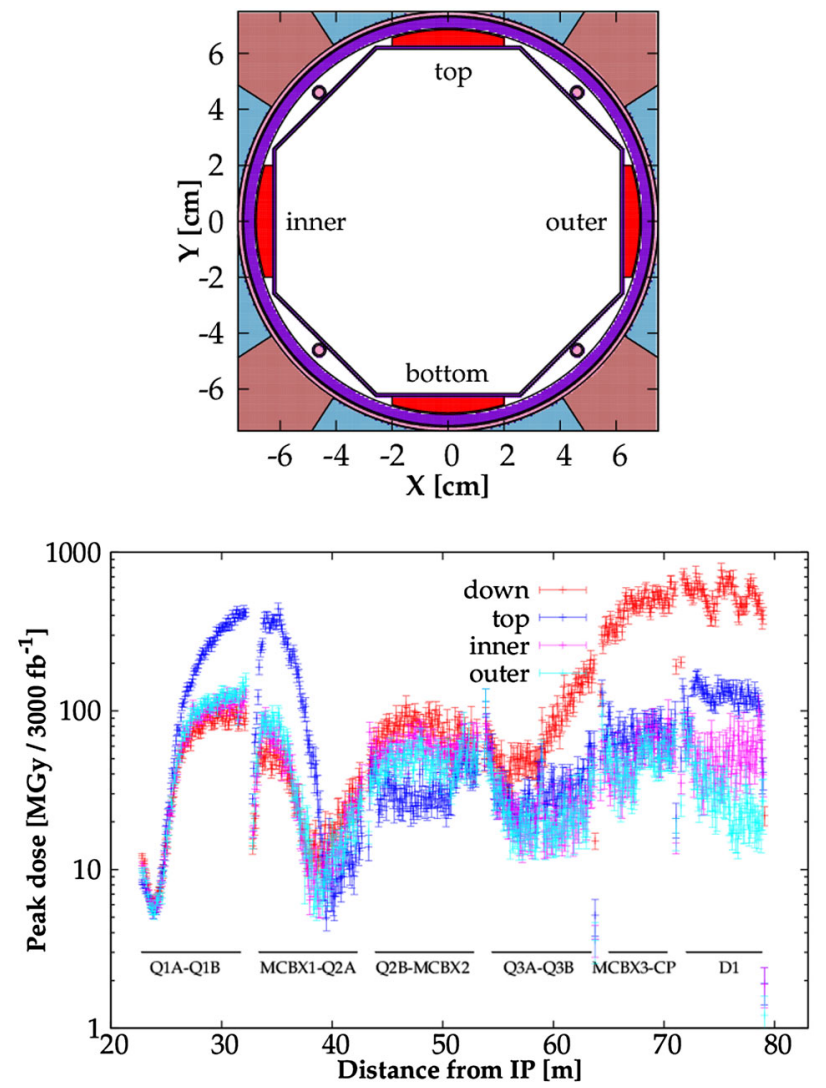

FIG. 10. Longitudinal peak dose profile (bottom) on different segments of the beam screen (top). 
TABLE II. Predicted radiation load (MGy) to organic materials in hottest components of the inner triplet.

\begin{tabular}{lcccr}
\hline \hline Component & Common name & Material & $\begin{array}{c}\text { Maximum calculated value } \\
\text { per } I_{0}=3000 \mathrm{fb}^{-1}\end{array}$ & Limit \\
\hline Q2B & Insulation & Kapton & 34 & $25-35$ \\
Q2B & Insulation & G10 & 25 & 20 \\
Q2B & Insulation/glue & Epoxy CTD-101 K & 24 & 25 \\
Q2B & Insulation & S2 fiberglass & 24 & 15 \\
Q2B & Insulation & G11 & 24 & $25-10$ \\
Q2B & Support material & Nomex & 6.7 & 15 \\
Q2B & Insulation & Polyimide & 6.7 & 25 \\
MCBX3 & Insulation & Kapton & 30 & $25-35$ \\
MCBX3 & Insulation/glue & Epoxy CTD-101 K & 27 & 25 \\
D1 & Insulation & Kapton & 22 & $25-35$ \\
D1 & Insulation & G10 & 20 & 20 \\
\hline \hline
\end{tabular}

see from Fig. 11 that a quite definite scaling is observed between the values of peak neutron fluence and peak DPA.

FLUKA and MARS results on neutron fluence are in quite good agreement. Results on DPA from two codes are also very similar in the $\mathrm{Q} 1 \mathrm{~A}$ through $\mathrm{Q} 3 \mathrm{~B}$ region with the MARS's values being slightly higher than those from FLUKA. At the same time discrepancy in DPA prediction increases in the opposite direction in D1, despite the consistency of neutron fluence values. Our attempts to explain this effect in the DPA behavior at the very end of the studied region have been unsuccessful so far.

The peak in the Q1B inner coil is about $2 \times 10^{-4} \mathrm{DPA}$ per $3000 \mathrm{fb}^{-1}$ integrated luminosity. In other IT components it is about $(1.0 \pm 0.5) \times 10^{-4}$. These numbers should be acceptable for the superconductors and copper stabilizer provided periodic annealing during the collider shutdowns. Taking into account a good correlation of DPA with neutron fluence in the coils, one can also compare the latter with the known limits. In the quadrupole coils, the peak fluence is $\sim 2 \times 10^{17} \mathrm{~cm}^{-2}$ which is substantially lower than the $3 \times 10^{18} \mathrm{~cm}^{-2}$ limit used for the $\mathrm{Nb}_{3} \mathrm{Sn}$ superconductor. In the orbit corrector and D1 dipole coils, the peak fluence is

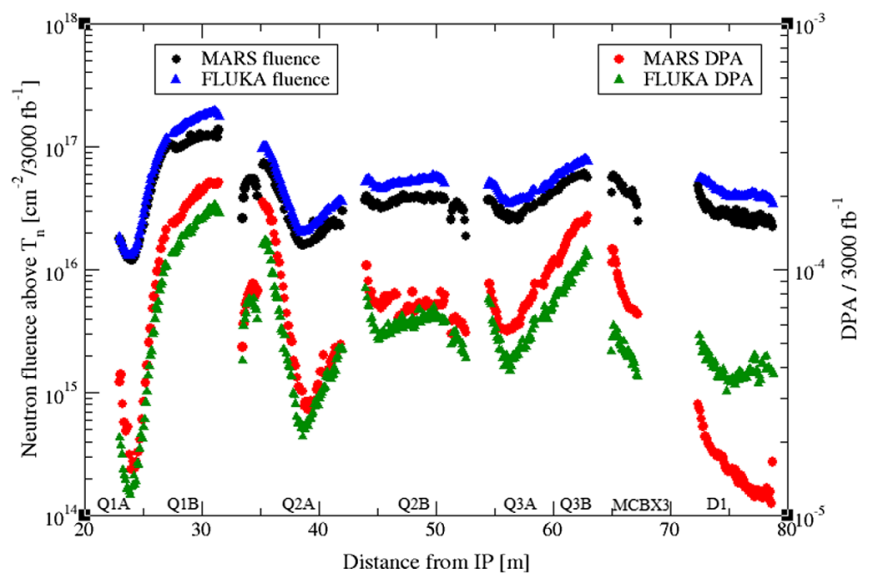

FIG. 11. Longitudinal peak neutron fluence and peak DPA profiles along the hottest regions in the IT magnet coils. $\sim 5 \times 10^{16} \mathrm{~cm}^{-2}$ which is again lower than the $10^{18} \mathrm{~cm}^{-2}$ limit used for the NbTi superconductor.

The integrated DPA in the magnet mechanical structures is 0.003 to 0.01 in the steel beam screen and tungsten absorber, $\sim 10^{-4}$ in the collar and yoke, and noticeably less outside. These are to be compared to a $10 \mathrm{DPA}$ limit for mechanical properties of these materials. Neutron fluences in the IT mechanical structures range from $3 \times 10^{16} \mathrm{~cm}^{-2}$ to $3 \times 10^{17} \mathrm{~cm}^{-2}$ compared to the $10^{21} \mathrm{~cm}^{-2}$ to $7 \times 10^{22} \mathrm{~cm}^{-2}$ limits.

\section{ENGINEERING CONSTRAINTS AND DESIGN EVOLUTION FOR BETTER SHIELDING}

The beam screen equipped with tungsten absorbers represents the backbone element for the protection of the IT magnets. Therefore, the details of its design play a crucial role in determining its actual effectiveness.

After the preliminary studies described in the previous section, new estimates were necessary to include: (i) the real absorber material, INERMET 180 that has a density of $18 \mathrm{~g} \mathrm{~cm}^{-3}$, about $8 \%$ less than pure tungsten, implying a reduced shielding performance; (ii) the first prototype drawing [24] that takes into account the machinability of INERMET and the required size of the cooling tubes as dictated by preliminary cryogenics estimates; (iii) the reduction of the beam screen thickness (from 2 to $1 \mathrm{~mm}$ ) necessary to let the structure respond elastically to possible deformations occurring during a quench.

Figure 12 (left) shows the transverse section of the beam screen model (BS\#2) embedding the aforementioned modifications. It can be compared to the model (BS\#1) used in the calculations reported in the previous section (see Figs. 3-6). The longitudinal peak dose profile on the inner coils of the IT magnets is presented in Fig. 13 for the case of BS\#1 (black points) and of BS\#2 (red points). In the latter case, the accumulated peak dose turns out to be systematically higher all along the IT magnets, almost doubling its value in the Q3 and reaching about 55 MGy in the MCBX3 corrector (however in an azimuthal position 

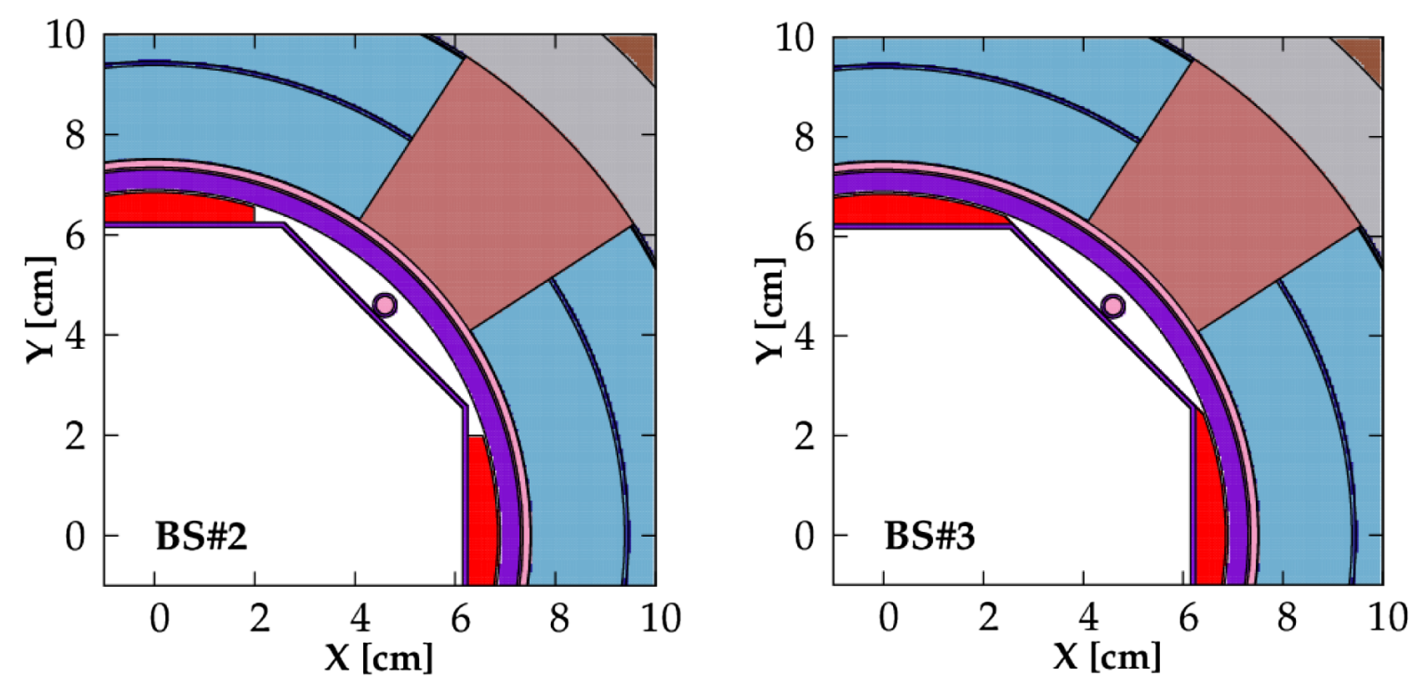

FIG. 12. Left: Beam screen model as per the first conceptual design (BS\#2) [24]. Right: Beam screen model with the modification of the absorbers driven by energy deposition considerations (BS\#3).

that in the MCBX design being detailed in the meantime lies in the collar outside the coil region). Along the Q2, most of the impacting debris, positively charged, is pushed by the magnetic field from the crossing angle side to the opposite one, i.e., from top to bottom in the assumed crossing scheme, where the outgoing beam points upwards. This moves the energy deposition peak through different azimuthal regions, which in the revised design (BS\#2) are no longer shielded by the beam screen absorbers, hence yielding the resulting substantial increase. In order to address this drawback, we considered a third version of the beam screen (BS\#3), where the INERMET absorbers were extended as much as possible to cover the coils towards the poles (see Fig. 12, right). The estimated peak dose distribution (green points in Fig. 13) shows a significant improvement in the Q3-MCBX3 region, when compared to the BS\#2 case. It should also be noted that, mainly due to the reduced absorber density, the sharing

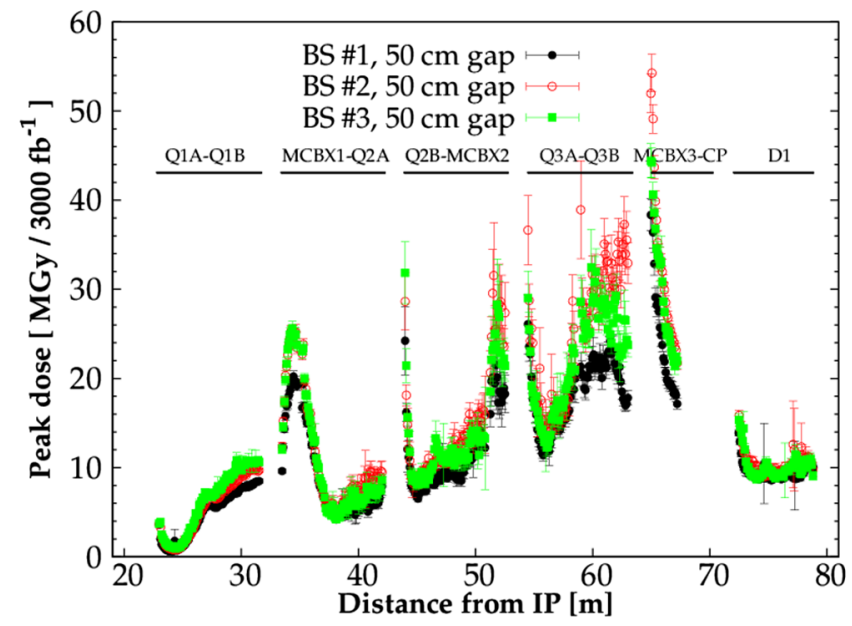

FIG. 13. Longitudinal distributions of peak dose on the inner coils of the IT magnets referring to different beam screen designs. of the total deposited power between the cold mass and beam screen gets unbalanced, moving to 55-45 and making the heat released in the cold mass approach $700 \mathrm{~W}$ (at $5 \times 10^{34} \mathrm{~cm}^{-2} \mathrm{~s}^{-1}$ ) against $630 \mathrm{~W}$ for the BS\#1 configuration as reported in Sec. IV. This has to be taken into account with a suitable margin in the cryoplant design.

Another crucial aspect is the longitudinal interruption of the beam screen and its absorbers, which is necessary between two consecutive cryostats in order to host a bellow and a Beam Position Monitor (BPM). As mentioned in the previous section, we initially assumed a $500-\mathrm{mm}$ gap. Shorter gaps are possible if the BPMs are going to be equipped with absorber layers like the ones in the beam screen. To mimic this case, we looked at the effect of a 100-mm gap, which should be considered as the most optimistic case. The peak dose dependence on the gap length is presented in Fig. 14 where the improvement

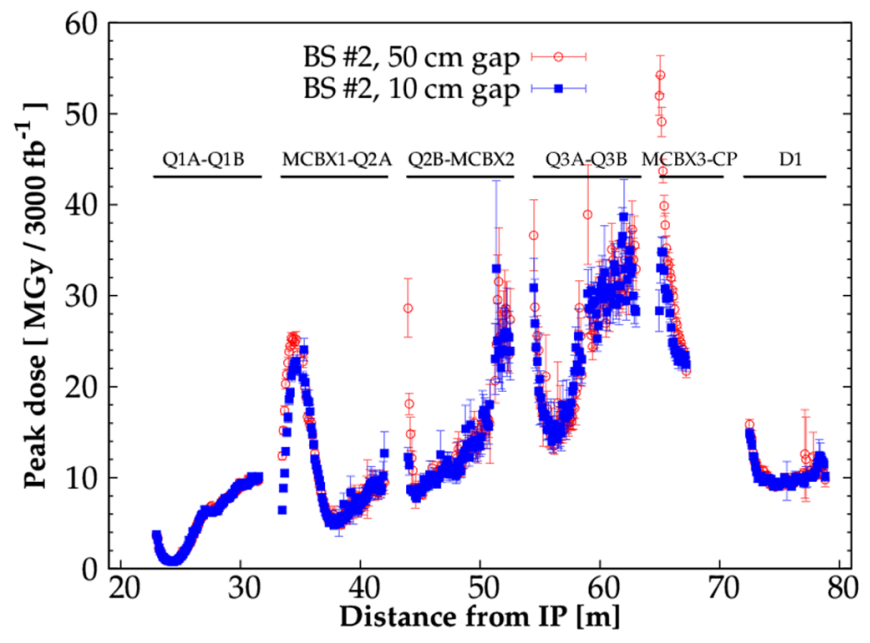

FIG. 14. Longitudinal distributions of peak dose on the inner coils of the IT magnets referring to different lengths of the beam screen gap in the interconnects. 
achieved downstream the Q2A-Q2B, MCBX2-Q3A and especially Q3B-MCBX3 interconnects is visible, with a reduction from 55 to $35 \mathrm{MGy}$ in the $\mathrm{MCBX} 3$ for the $\mathrm{BS} \# 2$ design. Therefore, the actual implementation of the absorber layers in the design of both the beam screen and the relevant BPMs considerably affects the maximum dose expected in the IT coils.

\section{SUMMARY}

It was shown that $80 \%$ of the energy released in pp-collisions leaks through the TAS apertures on the two sides of the experiments, resulting in $3.8 \mathrm{~kW}$ of dynamic heat load impacting the HL-LHC accelerator components on each side around IP1 and IP5. Very detailed descriptions of geometry, materials and magnetic fields for all the components in the inner triplet regions were implemented into the FLUKA and MARS 15 models to find the optimal parameters of the protective components needed to assure the operational performance of the IP1/IP5 150-mm aperture $\mathrm{Nb}_{3} \mathrm{Sn}$ final focus quadrupoles along with the $150-\mathrm{mm}$ aperture $\mathrm{NbTi}$ D1 separation dipoles and corrector magnets. Results of simulations with the two independent codes were found to be in good agreement. It was demonstrated that the proposed system of the tungsten-based inner absorbers assures the quench stability of the IT magnets with a safety margin close to or exceeding a factor of 10 as well as manageable dynamic heat loads on the IT cryogenic system. The peak DPA in nonorganic materials of the IT magnet coils is about $2 \times 10^{-4}$ DPA per $3000 \mathrm{fb}^{-1}$ integrated luminosity that should be acceptable for the superconductors and copper stabilizer provided annealing during the collider shutdowns. The peak dose accumulated in the magnet nonorganic materials at the same integrated luminosity is close to the established limits. To provide a reasonable safety margin, especially with respect to the ultimate goal of $4000 \mathrm{fb}^{-1}$, it implies that the limits need better understanding, advanced radiation-resistant materials should be considered (see, for example, Ref. [25]) and the ways to further improve protection efficiency of the inner absorber system need to be studied (e.g., a 100-mm gap in the beam screen and its absorbers rather than a $500-\mathrm{mm}$ one). Finally, whereas the study demonstrated the effectiveness of the conceptual design solution corroborated by a robust cross validation of the calculation tools, detailed numbers should not be taken as references carved in stone, since the actual design is still evolving, with the impact discussed in the previous section.

\section{ACKNOWLEDGMENTS}

This work was supported by Fermi Research Alliance, LLC under Contract No. DE-AC02-07CH11359 with the United States Department of Energy and by the HighLuminosity LHC Project.
[1] O. Brüning and L. Rossi, High luminosity Large Hadron Collider: A description for the European Strategy Preparatory Group, Report No. CERN ATS 2012-236.

[2] E. Todesco, H. Allain, G. Ambrosio, F. Borgnolutti, F. Cerutti, D. Dietderich, L. S. Esposito, H. Felice, P. Ferracin, G. Sabbi, P. Wanderer, and R. Van Weelderen, Design studies for the low-beta quadrupoles for the LHC luminosity upgrade, Report No. CERN ATS 2013-018.

[3] N. V. Mokhov and I. L. Rakhno, Mitigating radiation loads in Nb3Sn quadrupoles for LHC upgrades, Phys. Rev. ST Accel. Beams 9, 101001 (2006).

[4] N. Kimura, A. Yamamoto, T. Shintomi, A. Terashima, V. Kovachev, and M. Murakami, Heat transfer characteristics of Rutherford-type superconducting cables in pressurized He II, IEEE Trans. Appl. Supercond. 9, 1097 (1999).

[5] L. Chiesa, S. Feher, J. Kerby, M. Lamm, I. Novitski, D. Orris, J. P. Ozelis, T. J. Peterson, M. Tartaglia, and A. V. Zlobin, Thermal studies of a high gradient quadrupole magnet cooled with pressurized, stagnant superfluid, IEEE Trans. Appl. Supercond. 11, 1625 (2001).

[6] Composite Technology Development, Inc., Data-sheets.

[7] L. S. Esposito, F. Cerutti, and E. Todesco, FLUKA energy deposition studies for HL-LHC, in Proceedings of the 4th International Particle Accelerator Conference, IPAC2013, Shanghai, China, 2013 (JACoW, Shanghai, China, 2013), TUPFI021, pp. 1379-1381.

[8] S. Roesler, R. Engel, and J. Ranft, The Monte Carlo event generator DPMJET-III, in Proceedings of the Monte Carlo 2000 Conference, Lisbon, 2000 (Springer-Verlag Berlin, 2001), pp. 1033-1038.

[9] R. Aaij et al. (LHCb Collaboration), Measurement of the forward energy flow in pp collisions at $\sqrt{ } \bar{s}=7 \mathrm{TeV}$, Eur. Phys. J. C 73, 2421 (2013).

[10] A. Fedynitch, A revised hadronic interaction model for minimum bias interactions at LHC energies, Ph.D. thesis, CERN, 2015.

[11] F. Broggi, Energy deposition in the triplet and TAS issues, Proc. CARE-HHH-APD Workshop on Interaction Regions for the LHC Upgrade, DAFNE and SuperB (IR'07), https:// care-hhh.web.cern.ch/CARE-HHH/IR07/Proceedings/ IR07\%20Session\%205/S5-1-Broggi_IR07_A4.pdf.

[12] L. S. Esposito, F. Cerutti, A. Lechner, A. Mereghetti, V. Vlachoudis, and A. Patapenka, Power load from collision debris on the LHC Point 8 insertion magnets implied by the LHCb luminosity increase, in Proceedings of the 4th International Particle Accelerator Conference, IPAC-2013, Shanghai, China, 2013 (Ref. [7]), TUPFI022, pp. 1382-1384.

[13] A. Ferrari, P. Sala, A. Fasso, and J. Ranft, FLUKA, a multiparticle transport code, Report No. CERN-2005-010, 2005, http://www.fluka.org.

[14] T. T. Böhlen, F. Cerutti, M.P. W. Chin, A. Fassó, A. Ferrari, P. G. Ortega, A. Mairani, P. R. Sala, G. Smirnov, and V. Vlachoudis, The FLUKA code: Developments and challenges for high energy and medical applications, Nucl. Data Sheets 120, 211 (2014).

[15] N. V. Mokhov and C. James, The MARs Code System User's Guide, http://www-ap.fnal.gov/MARS/.

[16] N. V. Mokhov and S. I. Striganov, MARS15 overview AIP Conf. Proc. 896, 50 (2007). 
[17] N. Mokhov, P. Aarnio, Y. Eidelman, K. Gudima, A. Konobeev, V. Pronskikh, I. Rakhno, S. Striganov, and I. Tropin, MARS15 code developments driven by the intensity frontier needs, Prog. Nucl. Sci. Technol. 4, 496 (2014).

[18] C. Hoa, F. Cerutti, and E. Wildner, Energy deposition in the LHC insertion regions IR1 and IR5, LHC Project Report No. 1167, 2008.

[19] A. V. Zlobin, E. Barzi, D. Chichili, Yu. Huang, V. V. Kashikhin, M. Lamm, P. J. Limon, N. Mokhov, I. Novitski, T. Peterson, J. B. Strait, and S. Yadav, Large-aperture $\mathrm{Nb}_{3} \mathrm{Sn}$ quadrupoles for 2nd generation LHC IRs, in Proceedings of the 8th European Particle Accelerator Conference, Paris, 2002 (EPS-IGA and CERN, Geneva, 2002), MOPLE017, pp. 2451-2453.

[20] I. Novitski and A. V. Zlobin, Thermal analysis of SC quadrupoles in accelerator interaction regions, IEEE Trans. Appl. Supercond. 17, 1059 (2007).

[21] L. Bottura and P. Fessia, What could stop us, when and how long, RLIUP Proceedings, https://indico.cern.ch/ event/260492/session/0/contribution/3; K. Humer, H. W. Weber, E. K. Tschegg, S. Egusa, R. C. Birtcher, and H. Gerstenberg, Tensile and shear fracture behavior of fiber reinforced plastics at $77 \mathrm{~K}$ irradiated by various radiation sources, Adv. Cryog. Eng. 40, (1993).
[22] Synchrotron radiation from proton bunches creates photoelectrons at the beam screen wall. These photoelectrons are pulled toward the positively charged proton bunch. When they hit the opposite wall, they generate secondary electrons which can in turn be accelerated by the next bunch. Depending on several assumptions about surface reflectivity, photoelectron and secondary electron yield, this mechanism can lead to the fast buildup of an electron cloud with potential implications for beam stability and heat load on the beam screen, http://ab-abp-rlc.web.cern.ch/ ab-abp-rlc-ecloud/.

[23] R. E. MacFarlane and A. C. Kahler, Methods for processing ENDF/B-VII with NJOY, Nucl. Data Sheets 111, 2739 (2010), http://www.sciencedirect.com.

[24] R. Kersevan (private communication).

[25] A. V. Zlobin, G. Ambrosio, N. Andreev, E. Barzi, R. Bossert, G. Chlachidze, V. V. Kashikhin, S. Krave, F. Nobrega, and I. Novitski, Test results of $\mathrm{a} \mathrm{Nb}_{3} \mathrm{Sn}$ quadrupole coil impregnated with radiation-resistant Matrimid 5292, in Proceedings of the 4th International Particle Accelerator Conference, IPAC-2013, Shanghai, China, 2013 (Ref. [7]), pp. 3612-3614. 